\title{
PRINCIPAL CONGRUENCES OF $p$-ALGEBRAS AND DOUBLE $p$-ALGEBRAS
}

\author{
T. HECHT AND T. KATRIŇÁK
}

\begin{abstract}
Principal congruence of pseudocomplemented lattices $(=p-$ algebras) and of double pseudocomplemented lattices ( $=$ double $p$-algebras), i.e. pseudocomplemented and dual pseudocomplemented ones, are characterized.
\end{abstract}

1. Introduction. Recently H. Lakser [7] proved that every principal congruence of a distributive $p$-algebra is a join of two principal lattice congruences. We shall extend this result to all $p$-algebras (Theorem 1 ). The situation changes radically if one examines the double $p$-algebras. By Theorem 2, every principal congruence of a double $p$-algebra is a join of countably many principal lattice congruences. There exists even a distributive double $p$-algebra having a principal congruence which cannot be represented as a join of finite principal lattice congruences (Lemmas 3,4 and Example). In Theorem 3 we give a necessary and sufficient condition in order that every principal congruence of a double $p$-algebra be a join of finite principal lattice congruences.

2. Preliminaries. A universal algebra $\left\langle L ; \vee, \wedge,{ }^{*}, 0,1\right\rangle$ of type $\langle 2,2,1,0,0\rangle$ is called a $p$-algebra iff $\langle L ; \vee, \wedge, 0,1\rangle$ is a bounded lattice such that for every $a \in L$ the element $a^{*} \in L$ is the pseudocomplement of $a$, i.e. $x \leqslant a^{*}$ iff $a \wedge x=0$. A universal algebra $\left\langle L ; \vee, \wedge,{ }^{*},{ }^{+}, 0,1\right\rangle$ of type $\langle 2,2,1,1,0,0\rangle$ is called a double $p$-algebra iff $\left\langle L ; \vee, \wedge,{ }^{*}, 0,1\right\rangle$ is a $p$-algebra and $\langle L ; \vee, \wedge$, $\left.{ }^{+}, 0,1\right\rangle$ is a dual $p$-algebra $\left(x \geqslant a^{+}\right.$iff $\left.x \vee a=1\right)$. The standard results on $p$ algebras may be found in [3].

For a $p$-algebra $L$, define the set $B(L)=\left\{x \in L: x=x^{* *}\right\}$ of closed elements. The partial ordering of $L$ partially orders $B(L)$ and makes the latter into a Boolean algebra $\left\langle B(L) ; \cup, \wedge,{ }^{*}, 0,1\right\rangle$ for which $a \cup b=(a \vee b)^{* *}$ holds.

For any pair $a, b \in L$ in a $p$-, dual $p$-, or double $p$-algebra $L, \theta(a, b)$ denotes the principal congruence relation generated by $a, b$, i.e. the least congruence relation $\theta$ of this algebra for which $a \equiv b(\theta)$ is true. Clearly

$$
\theta(a, b)=\theta(a \wedge b, a \vee b) ;
$$

thus we need only characterize $\theta(a, b)$ for comparable $a, b$. We denote by

Received by the editors May 31, 1974.

AMS (MOS) subject classifications (1970). Primary 06A25, 06A35; Secondary 06A40.

Key words and phrases. $p$-algebra, dual p-algebra, double $p$-algebra, distributive $p$-algebra, distributive double $p$-algebra, Boolean algebra, pseudocomplement, dual pseudocomplement, closed element, principal congruence, principal lattice congruence.

C. American Mathematical Society 1976 
$\theta_{\text {Lat }}(a, b)$ the principal lattice congruence generated by $a, b ; \theta_{\text {Lat }}(a, b)$ has the substitution property for $\wedge$ and $\vee$, but not necessarily for ${ }^{*}$ or ${ }^{+}$.

For the definition of a unary algebraic function see [2]. By a unary lattice function we mean such a unary algebraic function which can be obtained from a lattice polynomial (see also [3]).

\section{Principal congruences of $p$-algebras.}

Lemma 1. Let $L$ be a p-algebra. Let $a, b \in L$ and $a \leqslant b$. If $p(x)$ is a unary lattice function on $L$ then the following identities hold:

(i) $p(a)^{*} \wedge a^{* *}=p(b)^{*} \wedge a^{* *}$;

(ii) $p(a)^{*} \wedge b^{*}=p(b)^{*} \wedge b^{*}$.

Proof. We proceed by induction on the rank of the lattice polynomial $r\left(x_{0}, \ldots, x_{n-1}\right)$, where $r\left(x, c_{1}, \ldots, c_{n-1}\right)=p(x), c_{1}, \ldots, c_{n-1} \in L$. If $p(x)$ is the identity or constant function (i) and (ii) hold trivially. Examine $p(x)$ $=q(x) \wedge t(x), q(x)$ and $t(x)$ satisfying (i). Then

$$
\begin{aligned}
p(a)^{*} \wedge a^{* *} & =[q(a) \wedge t(a)]^{*} \wedge a^{* *}=\left(q(a)^{*} \wedge a^{* *}\right) \cup\left(t(a)^{*} \wedge a^{* *}\right) \\
& =\left(q(b)^{*} \wedge a^{* *}\right) \cup\left(t(b)^{*} \wedge a^{* *}\right)=p(b)^{*} \wedge a^{* *} .
\end{aligned}
$$

Similarly, if $p(x)=q(x) \vee t(x)$ and $q(x)$ and $t(x)$ satisfy (i), then

$$
\begin{aligned}
p(a)^{*} \wedge a^{* *} & =[q(a) \vee t(a)]^{*} \wedge a^{* *}=q(a)^{*} \wedge t(a)^{*} \wedge a^{* *} \\
& =q(b)^{*} \wedge t(b)^{*} \wedge a^{* *}=p(b)^{*} \wedge a^{* *} .
\end{aligned}
$$

Thus we have proved property (i). The proof of (ii) is similar.

LemMa 2. Let $L$ be a lattice with 1 and let $d \in L$. We define a binary relation $\theta_{d}$ on $L$ in the following way:

$$
x \equiv y\left(\theta_{d}\right) \quad \text { iff } x \wedge d=y \wedge d .
$$

Then $\theta_{d} \leqslant \theta_{\text {Lat }}(d, 1)$.

The proof is straightforward.

TheOREM 1. Let $L$ be a p-algebra, let $a, b \in L$ and let $a \leqslant b$. Then

$$
\theta(a, b)=\theta_{\text {Lat }}(a, b) \vee \theta_{\text {Lat }}\left(\left(a^{*} \wedge b\right)^{*}, 1\right) .
$$

Proof. Let $\theta$ denote the lattice congruence on the right-hand side of (1). First we show that $\theta$ has the substitution property with respect to the operation

*. Let $x \equiv y(\theta)$. Then there is a sequence $x=z_{0}, \ldots, z_{n}=y$ of elements of $L$ and a sequence $p_{0}, \ldots, p_{n-1}$ of unary lattice functions such that

(I) $\left\{z_{i}, z_{i+1}\right\}=\left\{p_{i}(a), p_{i}(b)\right\}$ or

(II) $\left\{z_{i}, z_{i+1}\right\}=\left\{p_{i}\left(\left(a^{*} \wedge b\right)^{*}\right), p_{i}(1)\right\}$ for any $i=0,1, \ldots, n-1$ holds. Consider case (I). By Lemma 1, we have

$$
z_{i}^{*} \wedge a^{* *}=z_{i+1}^{*} \wedge a^{* *}, \quad z_{i}^{*} \wedge b^{*}=z_{i+1}^{*} \wedge b^{*} .
$$

Since $B(L)$ is a Boolean algebra, we get 


$$
z_{i}^{*} \wedge\left(a^{* *} \cup b^{*}\right)=z_{i+1}^{*} \wedge\left(a^{* *} \cup b^{*}\right) .
$$

By Lemma 2, the last identity implies $z_{i}^{*} \equiv z_{i+1}^{*}\left(\theta_{\text {Lat }}\left(\left(a^{*} \wedge b\right)^{*}, 1\right)\right)$, because $a^{* *} \cup b^{*}=\left(a^{*} \wedge b\right)^{*}$. In case (II) we obtain

$$
z_{i}^{*} \wedge\left(a^{*} \wedge b\right)^{*}=z_{i+1}^{*} \wedge\left(a^{*} \wedge b\right)^{*},
$$

by Lemma 1(i), bearing in mind $\left(a^{*} \wedge b\right)^{*} \in B(L)$. This implies $z_{i}^{*}$ $\equiv z_{i+1}^{*}\left(\theta_{\text {Lat }}\left(\left(a^{*} \wedge b\right)^{*}, 1\right)\right)$ by Lemma 2 . So, $x^{*} \equiv y^{*}(\theta)$ and $\theta$ is a ${ }^{*}$ congruence of $L$. Evidently $\theta(a, b) \leqslant \theta$. Conversely, $a \equiv b(\theta(a, b))$ yields $a^{*} \wedge b \equiv 0(\theta(a, b))$, and hence $\left(a^{*} \wedge b\right)^{*} \equiv 1(\theta(a, b))$. Thus, $\theta(a, b) \geqslant \theta$. Concluding, $\theta(a, b)=\theta$.

Corollary 1. Let $L$ be a p-algebra. Then $\theta(a, 1)=\theta_{\text {Lat }}(a, 1)$ for every $a \in L$.

Corollary 2. Let $L$ be a dual p-algebra, let $a, b \in L$ and let $a \leqslant b$. Then

$$
\theta(a, b)=\theta_{\text {Lat }}(a, b) \vee \theta_{\text {Lat }}\left(\left(a \vee b^{+}\right)^{+}, 0\right) .
$$

Corollary 3. Let $L$ be a dual p-algebra. Then $\theta(0, a)=\theta_{\text {Lat }}(0, a)$ for every $a \in L$.

REMARK 1. The analogue of Theorem 1 is also valid for the pseudocomplemented semilattices. (The proof of Theorem 1 is based on the fact that $B(L)$ is a Boolean algebra.)

REMARK 2. Theorem 1 was proved in [7] for the distributive p-algebras. In [4], an equivalent version of Theorem 1 has been proved for the modular $S$ algebras.

4. Principal congruences of double $p$-algebras. Let $L$ be a double $p$-algebra, let $x \in L$. We define $x^{n(+*)} \in L$ in the following way: $x^{1(+*)}=x^{+*}$, $x^{(k+1)(+*)}=x^{k(+*)+*}$ for every $k \geqslant 1$. Similarly we define $x^{n(*+)} \in L$. Since $a^{*} \vee a^{*+}=1$ implies $a^{* *} \wedge a^{*+*}=0$, we obtain $a^{*+*} \leqslant a^{*}$ in $L$. Therefore,

$$
x^{*} \geqslant x^{*+*} \geqslant \cdots \geqslant x^{* n(+*)} \geqslant \cdots
$$

in $L$. Dually we have

$$
y^{+} \leqslant y^{+*+} \leqslant \cdots \leqslant y^{+n(*+)} \leqslant \cdots
$$

for any $y \in L$.

Theorem 2. Let $L$ be a double p-algebra, let $a, b \in L$ and let $a \leqslant b$. Then

$$
\begin{aligned}
\theta(a, b)= & \theta_{\text {Lat }}(a, b) \\
& \vee \bigvee_{n \geqslant 0}\left[\theta_{\text {Lat }}\left(\left(a^{*} \wedge b\right)^{* n(+*)}, 1\right) \vee \theta_{\text {Lat }}\left(0,\left(a \vee b^{+}\right)^{+n(*+)}\right)\right] .
\end{aligned}
$$

Proof. Let $\theta$ denote the lattice congruence on the right-hand side of (5). It is a routine to show that $\theta(a, b) \geqslant \theta$. To conclude the proof we need only to show that $\theta$ has the substitution property with respect to the operations ${ }^{*}$ and 
+ . First we prove that $\theta$ is a ${ }^{*}$-congruence. Let $x \equiv y(\theta)$. Then there is a sequence $x=z_{0}, \ldots, z_{n}=y$ of elements of $L$ and congruences $\theta_{0}, \ldots, \theta_{n-1}$ such that $z_{i} \equiv z_{i+1}\left(\theta_{i}\right)$ where

(1) $\theta_{i}=\theta_{\text {Lat }}(a, b)$ or

(2) $\theta_{i}=\theta_{\text {Lat }}\left(\left(a^{*} \wedge b\right)^{* k(+*)}\right.$, 1) for some $k \geqslant 0$ or

(3) $\theta_{i}=\theta_{\text {Lat }}\left(0,\left(a \vee b^{+}\right)^{+m(*+)}\right)$ for some $m \geqslant 0$, and for any $i=0, \ldots$, $n-1$.

(1) $z_{i} \equiv z_{i+1}\left(\theta_{\text {Lat }}(a, b)\right)$ implies

$$
z_{i} \equiv z_{i+1}\left(\theta_{\text {Lat }}(a, b) \vee \theta_{\text {Lat }}\left(\left(a^{*} \wedge b\right)^{*}, 1\right)\right),
$$

by Theorem 1 . Therefore $z_{i}^{*} \equiv z_{i+1}^{*}(\theta)$.

(2) $z_{i} \equiv z_{i+1}\left(\theta_{\text {Lat }}\left(\left(a^{*} \wedge b\right)^{* k(+*)}, 1\right)\right)$ implies

$$
z_{i}^{*} \equiv z_{i+1}^{*}\left(\theta_{\text {Lat }}\left(\left(a^{*} \wedge b\right)^{* k(+*)}, 1\right)\right),
$$

by Corollary 1 to Theorem 1 . Therefore $z_{i}^{*} \equiv z_{i+1}^{*}(\theta)$.

(3) $z_{i} \equiv z_{i+1}\left(\theta_{\text {Lat }}\left(0,\left(a \vee b^{+}\right)^{+m(*+)}\right)\right)$ implies

$$
z_{i}^{*} \equiv z_{i+1}^{*}\left(\theta_{\text {Lat }}\left(0,\left(a \vee b^{+}\right)^{+m(*+)}\right) \vee \theta_{\text {Lat }}\left(\left(a \vee b^{+}\right)^{+m(*+) *}, 1\right)\right),
$$

by Theorem 1. Since $\left(a \vee b^{+}\right)^{+(m+1)(*+)} \vee\left(a \vee b^{+}\right)^{+m(*+) *}=1$, we have

$$
\theta_{\text {Lat }}\left(0,\left(a \vee b^{+}\right)^{+(m+1)(*+)}\right) \geqslant \theta_{\text {Lat }}\left(\left(a \vee b^{+}\right)^{+m(*+) *}, 1\right) \text {. }
$$

Therefore $z_{i}^{*} \equiv z_{i+1}^{*}(\theta)$.

Thus, $z_{i}^{*} \equiv z_{i+1}^{*}(\theta)$ for any $i=0, \ldots, n-1$, and we have proved $x^{*}$ $\equiv y^{*}(\theta)$, i.e. $\theta$ is a ${ }^{*}$-congruence of $L$. Using Corollaries 2 and 3 to Theorem 1 one can similarly prove that $\theta$ is a ${ }^{+}$-congruence of $L$, and so the proof is complete.

Corollary 1. Let $L$ be a double p-algebra, $a \in L$. Then

$$
\begin{aligned}
& \theta(a, 1)=\bigvee_{n \geqslant 0} \theta_{\text {Lat }}\left(0, a^{+n(*+)}\right) ; \\
& \theta(0, a)=\bigvee_{n \geqslant 0} \theta_{\text {Lat }}\left(a^{* n(+*)}, 1\right) .
\end{aligned}
$$

Proof. We know that $\theta_{\text {Lat }}(a, 1) \leqslant \theta_{\text {Lat }}\left(0, a^{+}\right)$and $\theta_{\text {Lat }}\left(a^{* * n}(+*), 1\right)$ $\leqslant \theta_{\text {Lat }}\left(a^{n(+*)}, 1\right) \leqslant \theta_{\text {Lat }}\left(0, a^{+n(*+)}\right)$ is true. Hence, by Theorem 2 , we have (6). By dual arguments we can prove (7).

COROllary 2. Let L be a double p-algebra in which the chains (3) and (4) are finite for every $x, y \in L$. Let $a, b \in L$ with $a \leqslant b$. Let $m$ be the least number with the property $\left(a^{*} \wedge b\right)^{* m(+*)}=\left(a^{*} \wedge b\right)^{*(m+1)(+*)}$ and $\left(a \vee b^{+}\right)^{+m(*+)}$ $=\left(a \vee b^{+}\right)^{+(m+1)(*+)}$. Then

$$
\begin{aligned}
\theta(a, b)= & \theta_{\mathrm{Lat}}(a, b) \vee \theta_{\mathrm{Lat}}\left(\left(a^{*} \wedge b\right)^{* m(+*)}, 1\right) \\
& \vee \theta_{\mathrm{Lat}}\left(0,\left(a \vee b^{+}\right)^{+m(*+)}\right) .
\end{aligned}
$$


COROllary 3. Let $L$ be a distributive double p-algebra satisfying the identities

$$
x^{* m(+*)}=x^{*(m+1)(+*)}, \quad x^{+m(*+)}=x^{+(m+1)(*+)}
$$

for some $m \geqslant 0$. Let $a, b \in L$ with $a \leqslant b$. If $x, y \in L$, then $x \equiv y(\theta(a, b))$ iff

$$
\begin{aligned}
& {\left[x \wedge a \wedge\left(a^{*} \wedge b\right)^{* m(+*)}\right] \vee\left(a \vee b^{+}\right)^{+m(*+)}} \\
& \quad=\left[y \wedge a \wedge\left(a^{*} \wedge b\right)^{* m(+*)}\right] \vee\left(a \vee b^{+}\right)^{+m(*+)}
\end{aligned}
$$

and

$$
\begin{aligned}
{\left[(x \vee b) \wedge\left(a^{*} \wedge b\right)^{* m(+*)}\right] \vee\left(a \vee b^{+}\right)^{+m(*+)} } \\
\quad=\left[(y \vee b) \wedge(a * \wedge b)^{* m(+*)}\right] \vee\left(a \vee b^{+}\right)^{+m(*+)}
\end{aligned}
$$

Proof. The proof follows from Corollary 2 and the fact that in a bounded distributive lattice $L$, for any elements $a_{1}, b_{1}, a_{2}, b_{2} \in L$ with $a_{1} \leqslant b_{1}$, the following statement is true:

$$
x \equiv y\left(\theta_{\text {Lat }}\left(a_{1}, b_{1}\right) \vee \theta_{\text {Lat }}\left(a_{2}, 1\right) \vee \theta_{\text {Lat }}\left(0, b_{2}\right)\right)
$$

iff

$$
\left(x \wedge a_{1} \wedge a_{2}\right) \vee b_{2}=\left(y \wedge a_{1} \wedge a_{2}\right) \vee b_{2}
$$

and

$$
\left[\left(x \vee b_{1}\right) \wedge a_{2}\right] \vee b_{2}=\left[\left(y \vee b_{1}\right) \wedge a_{2}\right] \vee b_{2}
$$

hold (see [3]).

RemarK. Corollary 3 combined with the result of A. Day [1] says that the equational subclass of the class of all distributive double $p$-algebras determined by the identities from Corollary 3 enjoys the Congruence Extension Property. We note here that the whole class of distributive double $p$-algebras has CEP (see [5]). Corollary 3 solves partially the problem mentioned in [5].

5. Counterexample. In this part we shall construct a distributive double $p$ algebra having a principal congruence which cannot be represented as a join of finite principal lattice congruences.

LeMma 3. Let $L$ be a double p-algebra. If $a \in L$ and $a^{* n(+*)}$ $>a^{*(n+1)(+*)}\left(a^{+n(*+)}<a^{+(n+1)(*+)}\right)$ for every integer $n \geqslant 0$ then $\theta(0, a)$ $(\theta(a, 1))$ cannot be represented as a join of finite principal lattice congruences of $L$.

Proof. Let $a^{* n(+*)}>a^{*(n+1)(+*)}$ for any $n \geqslant 0$. Suppose to the contrary that $\theta(0, a)$ is a join of finite principal lattice congruences of $L$. Then $\theta(0, a)$ is a compact element of the lattice of all lattice congruences on $L$ (cf. [2]). Therefore, by (3) and (6), there exists an integer $k \geqslant 0$ such that

$$
\theta(0, a)=\bigvee_{n=0}^{k} \theta_{\text {Lat }}\left(a^{* n(+*)}, 1\right)=\theta_{\text {Lat }}\left(a^{* k(+*)}, 1\right) .
$$


Evidently $a^{*(k+1)(+*)} \equiv 1(\theta(0, a))$. On the other hand,

$$
a^{*(k+1)(+*)} \not \equiv 1\left(\theta_{\text {Lat }}\left(a^{* k(+*)}, 1\right)\right)
$$

(see [3]), a contradiction. The second part can be proved dually.

Lemma 4. Let $B$ be a Boolean algebra, let $\varphi: B \rightarrow B$ be $a\{0,1, \wedge\}$ homomorphism and let $\psi: B \rightarrow B$ be $a\{0,1, \vee\}$-homomorphism such that $a \varphi \psi \leqslant a$ and $a \psi \varphi \geqslant a$ for every $a \in B$. Then the set $L=\left\{(a, b) \in B^{2}: a \varphi\right.$ $\geqslant b\}$ is a $\{0,1\}$-sublattice of $B^{2}$ and, moreover, $L$ forms a distributive double $p$ algebra in which for $t=(a, b) \in L$,

$$
t^{*}=\left(a^{\prime}, a^{\prime} \varphi\right), \quad t^{+}=\left(b^{\prime} \psi, b^{\prime}\right)
$$

is true.

For the proof see [6, Theorem 2].

EXAMPLE. Let $B$ denote the Boolean algebra of all subsets of the set $N$ of positive integers. Set

$A \varphi=\{x \in N: x \in A$ and $x+1 \in A\}$ for every $A \in B$,

$A \psi=\{x \in N: x \in A$ or $x-1 \in A\}$ for every $A \in B$.

It is easy to verify that $\varphi$ is a $\{0,1, \wedge\}$-homomorphism of $B$ into $B, \psi$ is a $\{0,1, \vee\}$-homomorphism of $B$ into $B$, both of which satisfy $A \varphi \psi \leqslant A$ and $A \psi \varphi \geqslant A$ for every $A \in B$. Let $L=\left\{(X, Y) \in B^{2}: X \varphi \geqslant Y\right\}$ be the distributive double $p$-algebra (see Lemma 4). Let $K_{n}=\{1, \ldots, n\} \in B$. If we set $a=\left(N-K_{1}, N-K_{1}\right)$ and $b=a^{+}$then $a^{+}=b=\left(K_{2}, K_{1}\right), a^{+*}=(N$

$\left.-K_{2}, N-K_{2}\right), a^{+*+}=b^{*+}=\left(K_{3}, K_{2}\right)$. By induction it is easy to prove

$$
\begin{aligned}
& a^{+n(*+)}=\left(K_{n+2}, K_{n+1}\right), \\
& b^{* n(+*)}=\left(N-K_{n+2}, N-K_{n+2}\right) .
\end{aligned}
$$

Now we see that $b^{* n(+*)}>b^{*(n+1)(+*)}$ and $a^{+n(*+)}<a^{+(n+1)(*+)}$ for every integer $n \geqslant 0$. So, by Lemma 3, $\theta(a, 1)$ and $\theta(0, b)$ cannot be represented as a join of finite principal lattice congruences of $L$.

Concluding we obtain

Theorem 3. Let $L$ be a double p-algebra. Let $a, b \in L$. Then the principal congruence $\theta(a, b)$ is a join of finite principal lattice congruences of $L$ iff the chains (3) and (4) are finite for every $x, y \in L$.

The proof follows from Corollary 2 to Theorem 2 and Lemma 3.

\section{REFERENCES}

1. A. Day, A note on the congruence extension property, Algebra Universalis 1 (1971/72), 234-235. MR 45 \#3288.

2. G. Grätzer, Universal algebra, Van Nostrand, Princeton, N.J., 1968. MR 40 \#1320.

3. Lattice theory. First concepts and distributive lattices, Freeman, San Francisco, Calif., 1971. MR 48 \# 184.

4. T. Katriňák, Primitive Klassen von modularen S-Algebren, J. Reine Angew. Math. 261 (1973), 55-70. 
5. Congruence extension property for distributive double p-algebras, Algebra Univervalis 4 (1974), 273-276. MR 50 \#6953.

6. Construction of regular double p-algebras, Bull. Soc. Roy. Sci. Liège 43 (1974), 283-290.

7. H. Lakser, Principal congruences of pseudocomplemented distributive lattices, Proc. Amer. Math. Soc. 37 (1973), 32-36.

Katedra Algebry a Teórie Čísel PfUK, 81631 Bratislava 16, Mlynská Dolina, CzechOSLOVAKIA 